\section{Stability of Interstitial Elements in Metal Lattice Evaluated by Statistical Thermo- dynamics}

\section{Introduction}

It is known that statistical thermodynamics is a bridge spanning between microscopic (atomistic) materials parameters (e.g., magnitude of interaction energy $E_{i-j}$ between component atoms, $i$ and $j$, and mode of atomic clustering in $M X_{x}$ ) and macroscopic (phenomenological) materials behaviors (e.g., equilibrium P-T-C (pressure-temperature-composition) relationship). Nonetheless, probably due to apparent mathematical complexity of formulation, statistical thermodynamics does not seem to be widely employed among materials scientists compared with traditional thermodynamics. However, in actuality, we can reduce statistical thermodynamic formulae to a set of simplifying equations which are readily applicable to analysis of experimental $\mathrm{P}-\mathrm{T}-\mathrm{G}$ data for interstitial solution of $X$ (non-metal) in $M$ (metal), $M X_{x} \cdot{ }^{1-19)}$

One of merits of statistical thermodynamic approach is its feasibility to derive $E_{X-M}$ value which is directly referring to $X-M$ bond strength in $M X_{x}$ with common reference state of energy zero taken at each component atom at rest being in infinite separation in vacuum. Thence, the relative stability of the interstitial elements $X$ in the same metal $M$ might be easily compared.

Comparison of this nature is not feasible by thermodynamic means, since reference states for different interstitial elements are usually not identical. For example, as depicted in Fig. 1, $\Delta H_{f}\left(M X_{x}\right)$ refers to the difference in energy between the condensed phase $M X_{x}$ and their reference states, namely $1 \mathrm{~mol}$ of pure condensed phase of $M$ and $x / 2 \mathrm{~mol}$ of $X_{2}$ gas (in case that the stable state of element $X$ is diatomic $X_{2}$ gas). Thus, comparison between $\Delta H_{f}$ values for different elements $X$ would not provide any useful information on relative stability of concerned interstitial elements in one metal, while such comparison might be of help to judge the stability of a specific interstitial element $X$ in different metals.

In this report, some distinguishable features of statistical thermodynamic analysis from those of traditional thermodynamics are presented by summarizing integrally the results obtained by now. ${ }^{1-19}$ )

\section{Fundamental Statistical Thermodynamic Equations}

Statistical thermodynamic equilibrium condition for interstitial solution $M X_{x}$ might be summarized into following set of generalized equation $\mathrm{s}^{1-19)}$ :

$$
\begin{aligned}
& \mathrm{A} \equiv R T \ln \left\{[(\theta-x) / x] \cdot a_{X}\right\}=\mathbf{K}-\mu_{X}^{0}+\beta x E_{X-X} \\
& \mathrm{~K}=Q-R T \ln f_{X} \\
& \partial E / \partial n_{X}=Q+\beta x E_{X-X}
\end{aligned}
$$

where, $a_{X}$ : the activity of the element $X$

$E:$ the lattice energy of $M X_{x}$

$f_{X}$ : the atomic partition function of element $X$ in $M X_{x}$

$n_{x}$ : the number of component atoms $X$ in $M X_{x}$

$Q:$ the extent of stabilization of $X$ atom through formation of $X-M$ bonds in $M X_{x}$ with reference to isolated $X$ atom

$R$ : the universal gas constant

$T:$ the absolute temperature

$\beta$ : a factor determined by crystal structure consideration (e.g., $\beta=12$ for interstitial solution with $\mathrm{NaCl}$ type structure)

$\theta:$ the number of interstitial sites available for occupation by $X$ atoms per $M$ atom in $M X_{x}$

$\mu_{x}^{0}$ : the standard chemical potential of $X$ with reference state being taken as monoatomic $X$ at rest in vacuum.

Expressions of the parameters, $a_{X}$ and $\mu_{x}^{0}$, vary depending on the stable form of the element $X$ as summarized in Table 1.

In this line of analysis, a priori assumption of constant $E_{X-X}$ within a phase at a given $\mathcal{T}$ was taken and value of $\theta$ was chosen to satisfy this assumption. Validity of this assumption appeared to be supported by following features of estimate results $\mathrm{s}^{5,8,9,14)}$; values of $\theta$ are close to solubility limit of $X$ in $M X_{x}: Q$-values for the element $X$ in the metals in the same group in the periodic table are comparable to one another $(c f$.,

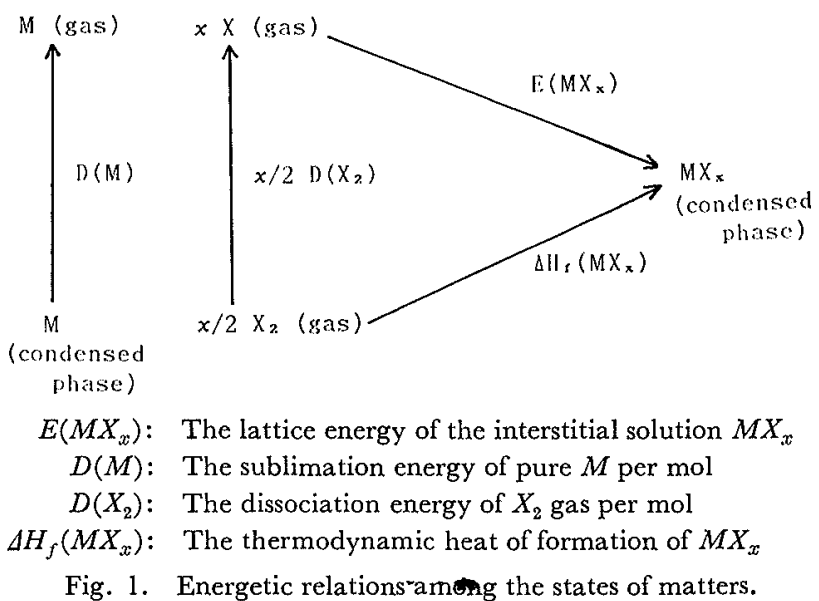


Table 1. Expressions of $a_{X}$ and $\mu_{X}^{0}$ for different interstitial elements.

\begin{tabular}{lllcc}
\hline \multicolumn{1}{c}{ Stable form of $X$} & $a_{X}$ & \multicolumn{1}{c}{$\mu_{X}^{0}$} & Example & Ref. \\
\hline Ideal diatomic gas $X_{2}$ & $\sqrt{p\left(X_{2}\right)}$ & $\begin{array}{c}-(1 / 2 D)\left(X_{2}\right)-(1 / 2) R T \ln \left\{\left[\left(4 \pi m_{X}\right)^{3 / 2}(k T)^{5 / 2} / h^{3}\right] \cdot\right. \\
\left.\left(T / \Theta_{\gamma}\right) \cdot\left[1+\left(\Theta_{Y} / 3 T\right)\right] \cdot\left(\rho^{2} / 2\right)\right\}+(1 / 4) R \Theta_{v}+(1 / 2) R T \\
\end{array}$ & $\begin{array}{l}\mathrm{H}_{2}, \mathrm{~N}_{2}, \mathrm{P}_{2} \\
\text { ln }\left[1-\exp \left(-\Theta_{v} / T\right)\right]\end{array}$ & $\begin{array}{c}1)-6), 8)-12), 14), \\
15), 17)-19)\end{array}$ \\
\hline Ideal monoatomic gas $X$ & $p(X)$ & $-R T \ln \left\{\left[\left(2 \pi m_{X}\right)^{3 / 2}(k T)^{5 / 2}\right] / h^{3}\right\}$ & $\mathrm{S}$ & $13)$ \\
\hline Pure condensed $X$ & $a_{X}$ & $-D(X)$ & $\mathrm{C}$ & $7), 11), 16)$ \\
\hline
\end{tabular}

$p\left(X_{2}\right), p(X)$ : Equilibrium pressure of $X_{2}$ gas and that of $X$ gas, respectively, $h$ : Planck's constant,

$k$ : Boltzmann's constant, $\quad m_{X}$ : Mass of $X$ atom, $\quad \theta_{r}$ : Characteristic temperature of rotation of $X_{2}$ molecule,

$\Theta_{v}$ : Characteristic temperature of vibration of $X_{2}$ molecule,

$\rho:$ Nuclear spin weight of $X$ atom

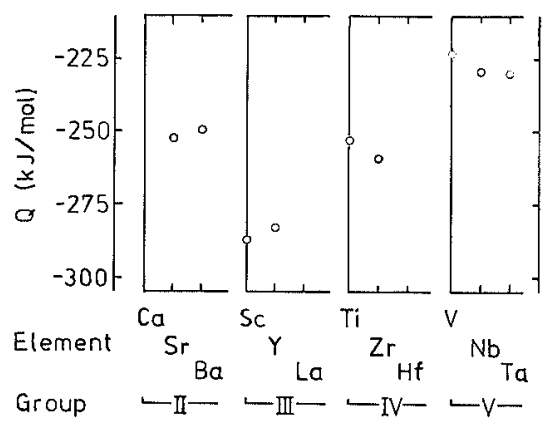

Fig. 2. Graphic presentation of $Q$-values for primary $\mathrm{H}$-solutions in II V-group metals estimated by statistical thermodynamics.

Fig. 2).

$$
\begin{aligned}
\text { II: } & M=\mathrm{Sr}, \mathrm{Ba}^{5)} \\
\text { III: } & M=\mathrm{Sc}, \mathrm{Y}^{9)} \\
\text { IV: } & M=\mathrm{Ti}, \mathrm{Zr}^{8)} \\
\mathrm{V}: & M=\mathrm{V}, \mathrm{Nb}, \mathrm{Ta}^{2,5)}
\end{aligned}
$$

\section{Analysis Results for $\operatorname{Fe} X_{x}(\boldsymbol{X}=\mathbf{H}, \mathbf{C}, \mathbf{N}, \mathbf{P}, \mathbf{S})$}

The results obtained from the analyses along this line for several interstitial solutions $\mathrm{Fe} X_{x}$ might be summarized as shown in Fig. 3 (see also Table 1 in Ref. 16)).

This figure appears to indicate clearly that the stability of the interstitial elements in Fe-lattice increases as the following order:

$$
\mathrm{H}<\mathrm{S}<\mathrm{P}<\mathrm{N}<\mathrm{C}
$$

Or, more specifically, it might be argued as follows:

(A) When compared for interstitial elements of 4 th to 6 th groups in the periodic table, there appears to be a trend that stability of the element in Fe-lattice decreases as group number increased.

(B) Through comparison for $\mathrm{N}$ and $\mathrm{P}$, it might be said that stability of the element in Fe-lattice tends to decrease as periodic number increased within the group.

It is accepted that the atomic radii of these interstitial elements increases as the following order:

$$
\mathrm{H}<\mathrm{N}<\mathrm{C}<\mathrm{P}<\mathrm{S}
$$

Provided that the elastic strain energy term is the decisive factor for the stability of these elements in Fe-lattice, $\mathrm{H}$ must be the most stable and $\mathrm{S}$ the least stable. However, as shown in Eq. (4), statistical thermodynamic evaluation suggests that $\mathrm{C}$ is the most stable in Fe-lattice and $\mathrm{H}$ the least stable. Therefore, it is evident that the decisive factor for the stability of these interstitial elements in Fe-lattice must

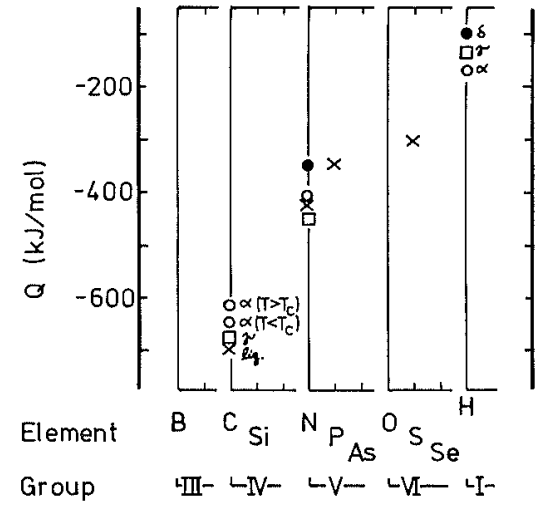

Fig. 3. Graphical presentation of $Q$-values for miscellaneous interstitial elements in Fe-lattice. ${ }^{11-13,15,16)}$

be the nature of electronic (chemical) interaction between $X$ atom and $\mathrm{Fe}$ atom in $\mathrm{Fe} X_{x}$ rather than the elastic strain energy term.

When comparison is made among the $Q$-values for different $\mathrm{FeN}_{x}$ phases, following features might be pointed out:

(C) $\mathrm{N}$ atom in fcc $\gamma-\mathrm{FeN}_{w}$ phase appears to be the most stable (Note that $\mathrm{H}$ in $\alpha-\mathrm{FeH}_{x}$ seems to be more stable than $\mathrm{H}$ in $\gamma-\mathrm{FeH}_{x}$ ).

(D) $\mathrm{N}$ atom in high temperature bcc phase ( $\delta$ phase) is less stable than that in low temperature bcc phase ( $\alpha$-phase).

(E) Stability of $\mathrm{N}$ atom in molten $\mathrm{FeN}_{x}$ is higher than that in bcc phases but lower than that in fcc phase (Note that $\mathrm{G}$ in molten $\mathrm{FeC}_{x}$ seems to be more stable than $\mathrm{G}$ in $\gamma-\mathrm{FeG}_{x}$ ).

These features do not appear to be incompatible with available experimental solubility data (e.g., Table 1 and Fig. 2 in Ref. 20) for N-solubility: Fig. 1 and Table 2 in Ref. 21) for H-solubility).

During the course of analysis of $\alpha-\mathrm{FeG}_{x},{ }^{16)}$ it was noticed that $Q$-value might be affected to an appreciable extent by the magnetic transition occurring at around Curie temperature $\left(T_{C} \sim 1000 \mathrm{~K}\right)$; i.e., $Q_{c}^{\alpha}(T<1000 \mathrm{~K})=-648 \mathrm{~kJ} / \mathrm{mol}$ and $Q_{c}^{\alpha}(1025 \mathrm{~K}<T<$ $1150 \mathrm{~K})=-613 \mathrm{~kJ} / \mathrm{mol}$. The observed trend of more negative $Q_{G}^{\alpha}$ at $I$ lower than $T_{C}$ than that at $T$ higher than $T_{C}$ appears to be in qualitative accord with earlier available analysis result (e.g., Ref. 22)). However, no such effect was discernible for $\alpha-\mathrm{FeH}_{x}$ and for $\alpha-\mathrm{FeN}_{x}$. Thus, further detailed examinations are desired to clarify this point. 


\section{Analysis of Ternary $M Z_{z} X_{x}$ Phase with Two Interstitial Constituents}

In the earlier works, $\mathrm{P}$ solubility in ternary molten $\mathrm{Fe} Z_{z} \mathrm{P}_{x} \quad(Z=\mathrm{B}, \mathrm{C})^{12)}$ and $\mathrm{S}$ solubility in molten $\left.\mathrm{Fe} Z_{z} \mathrm{~S}_{x}(Z=\mathrm{C}, \mathrm{P})^{13}\right)$ were briefly discussed. In these studies, it was concluded that $\theta$ available for occupation of $X$ atoms in $\mathrm{Fe} Z_{z} X_{x}(X=\mathrm{P}, \mathrm{S})$ might be expressed as follows:

$$
\theta\left(\mathrm{Fe} Z_{z} X_{x}\right)=\theta\left(\mathrm{Fe} X_{x}\right)-s z
$$

where, $s:$ a constant determined for combinations of elements, $X$ and $Z$ (e.g., $s=0.4$ for $Z=\mathrm{B}$ and $s=1.25$ for $Z=\mathrm{C}$ in molten $\mathrm{Fe}_{z} \mathrm{P}_{x}{ }^{12)}$ and $s=4$ for $Z=\mathrm{G}$ and $s=2.75$ for $Z=\mathrm{P}$ in molten $\mathrm{Fe} Z_{z} \mathrm{~S}_{x}{ }^{13)}$ ).

Eq. (6) suggests that solubility limit $\theta$ of element $X$ in molten $\mathrm{Fe} Z_{z} X_{x}$ tends to decrease from that in $\mathrm{Fe} X_{x}, \theta\left(\mathrm{Fe} X_{x}\right)$, as $z$ increased. Validity of $\mathrm{Eq}$. (6) was also appreciated as the evidence of negligible $E_{X-Z}$ in these molten $\mathrm{Fe}_{z} X_{x}$ phases discussed there. ${ }^{12,13)}$

With reference to the stability hierarchy of interstitial elements in Fe-lattice given as Eq. (4), it might be noticed that it is essential to take more stable interstitial element in Fe-lattice as $Z$ and less stable one as $X$ in deriving relation like Eq. (6). For example, in analyzing $\mathrm{FeC}_{z} \mathrm{P}_{x}$, it would be realistic to consider $\mathrm{FeC}_{z} \mathrm{P}_{x}$ as the solution of $\mathrm{P}$ in $\mathrm{FeC}_{z}$ possessing a fixed composition $z$, as was done in Ref. 12). However, it would not be practical, on the contrary, to consider $\mathrm{FeC}_{z} \mathrm{P}_{x}$ as the solution of $\mathrm{C}$ in $\mathrm{FeP}_{x}$ possessing a fixed composition $x$, since the stability of $\mathrm{C}$ in Fe-lattice is higher than that of $\mathrm{P}$ and, hence, by forced addition of $\mathrm{C}$ to $\mathrm{FeP}_{x}$ to form $\mathrm{FeC}_{z} \mathrm{P}_{x}, \mathrm{P}$ might be expelled from Fe-lattice and the composition $x$ might be shifted easily from its initial value.

Stability herarchy of interstitial elements as given by Eq. (4) might be of pragmatic use for planning purification processes of metals.

As such, statistical thermodynamics appears to be a useful analysis tool for ternary $M Z_{z} X_{x}$ with two interstitial components as well as for binary $M X_{x}$, leading to unambiguous definition for solubility limit through parameter $\theta$ and for stability of interstitial element through parameter $Q$ calculated on the basis of chosen $\theta$-value.

Re-constructed $\log p\left(\mathrm{P}_{2}\right)$ vs. $x$ relations for $\mathrm{FeC}_{z} \mathrm{P}_{x}{ }^{12)}$ and re-constructed $\log \left[p\left(\mathrm{H}_{2} \mathrm{~S}\right) / p\left(\mathrm{H}_{2}\right)\right]$ vs. $x$ relations for $\mathrm{FeC}_{z} \mathrm{~S}_{x}{ }^{13)}$ on the basis of estimated statistical thermodynamic parameters appeared to reproduce experimental $\mathrm{P}-\mathrm{T}-\mathrm{C}$ relations reasonably well.

\section{Concluding Remark}

Feasibility of direct comparison of relative stability among different interstitial elements in a metal lattice in terms of parameter $Q$ is one of attractive merits of statistical thermodynamics. Direct comparison of this nature is not feasible with thermodynamic parameters, while thermodynamics is a useful tool for comparing relative stability of certain interstitial element among several metal lattices and for analyzing substitutional solutions and stoichiometric compounds.

When ternary phase $M Z_{z} X_{x}$ with two interstitial constituents, $X$ and $Z$, is under consideration and $Z$ is known to be more stables than $X$ in $M$-lattice, it appears to be rational to consider $M Z_{z} X_{x}$ as the solution of $X$ in $M Z_{z}$ rather than the other way round.

In spite of the term statistical, discussion on the basis of statistical thermodynamics proved to be useful for clarifying ordering phenomena of interstitial elements in $M X_{x}{ }^{17,23)}$ In addition, careful analysis of variation of statistical thermodynamic parameter for $\mathrm{Fe}_{1-y} M_{y} \mathcal{N}_{x}$ with respect to $y^{15,18,19)}$ led straightforwardly to the model with definite clustering of metal atoms $\mathrm{Fe}_{6-n} M_{n}$ ( $n=1$ or 4 for $M=\mathrm{V}, \mathrm{Gr}$ depending on $y^{15,18)}$ and $n=4$ for $M=\mathrm{Ti}^{19)}$ ) surrounding an $\mathrm{N}$ atom on O-site. Such aspect of atomic clustering in alloys cannot be derived easily with traditional thermodynamic approach.

\section{REFERENCES}

1) N. Shohoji, M. Katsura and T. Sano: J. Less-Common Met., 38 (1974), 59.

2) N. Shohoji: J. Less-Common Met., 90 (1983), L27.

3) N. Shohoji: phys. stat. solid. (B), 118 (1983), 811.

4) N. Shohoji: phys. stat. solid. (B), 119 (1983), K87.

5) N. Shohoji: J. Less-Common Met., 102 (1984), 53.

6) N. Shohoji: Mater. Lett., 3 (1985), 206.

7) N. Shohoji: Mater. Chem. Phys., 15 (1985), 567.

8) N. Shohoji: Z. Matallkd., 76 (1985), 192.

9) N. Shohoji: J. Less-Common Met., 114 (1985), 249.

10) N. Shohoji: J. Nucl. Mater., 127 (1985), 88.

11) N. Shohoji: J. Mater. Sci., 21 (1986), 2147.

12) N. Shohoji: Trans. Iron Steel Inst. Jpn., 26 (1986), 194.

13) N. Shohoji: Trans. Iron Steel Inst. Jpn., 26 (1986), 547.

14) N. Shohoji: Surface Coat. Technol., 28 (1986), 365.

15) N. Shohoji: Mater. Sci. Technol., 3 (1987), 43.

16) N. Shohoji: Mater. Sci. Technol., 3 (1987), 404.

17) N. Shohoji and T. Marcelo: J. Mater. Sci. Lett., 6 (1987), 1251.

18) N. Shohoji: Mater. Sci. Technol., 4 (1988), 867.

19) N. Shohoji: Mater. Chem. Phys., 24 (1989), 163.

20) R. B. McLellan and R. J. Farraro: Acta metall., 28 (1980), 417.

21) J.R.G. da Silva and R. B. McLellan: J. Less-Common Met., 50 (1976), 1.

22) T. Nishizawa and M. Hasebe: Tetsu-to-Hagané, 67 (1981), 1887.

23) N. Shohoji and T. Marcelo: Mater. Chem. Phys., 16 (1987), 475.

(Received on November 16, 1989; accepted in the final form on February $16,1990)$

Nobumitsu SHOHOJI

Departamento de Tecnologia de Materiais,

Laboratório Nacional de Engenharia e Tecnologia Industrial, 1699 Lisboa Codex, Portugal. 